\title{
Performance and efficacy of 320-row computed tomography coronary angiography in patients presenting with acute chest pain: results from a clinical registry
}

\author{
J. E. van Velzen • F. R. de Graaf • L. J. Kroft • \\ A. de Roos $\cdot$ J. H. C. Reiber $\cdot$ J. J. Bax $\cdot$ J. W. Jukema $\cdot$ \\ J. D. Schuijf • M. J. Schalij • E. E. van der Wall
}

Received: 21 February 2011 / Accepted: 11 May 2011/Published online: 26 May 2011

(C) The Author(s) 2011. This article is published with open access at Springerlink.com

\begin{abstract}
The purpose of this study was to evaluate the performance of 320-row computed tomography angiography (CTA) in the identification of significant coronary artery disease (CAD) in patients presenting with acute chest pain and to examine the relation to outcome during follow-up. A total of 106 patients with acute chest pain underwent CTA to evaluate presence of CAD. Each CTA was classified as: normal, nonsignificant $\mathrm{CAD}(<50 \%$ luminal narrowing) and significant $\mathrm{CAD}$ ( $\geq 50 \%$ luminal narrowing). CTA results were compared with quantitative coronary angiography. After discharge, the following cardiovascular events were recorded: cardiac death, non-fatal infarction, and unstable angina requiring revascularization. Among the 106 patients, 23 patients (22\%) had a normal CTA, 19 patients (18\%) had non-significant CAD on CTA, 59 patients (55\%) had significant CAD
\end{abstract}

J. E. van Velzen · F. R. de Graaf · J.

J. Bax · J. W. Jukema · J. D. Schuijf · M.

J. Schalij · E. E. van der Wall ( $\square)$

Department of Cardiology, Leiden University Medical

Center, Postal zone: C5-P, P.O. Box 9600, 2333 ZA

Leiden, The Netherlands

e-mail: e.e.van_der_wall@lumc.nl

J. E. van Velzen · J. W. Jukema · E. E. van der Wall The Interuniversity Cardiology Institute of the Netherlands, Utrecht, The Netherlands

L. J. Kroft · A. de Roos · J. H. C. Reiber

Department of Radiology, Leiden University Medical

Center, Leiden, The Netherlands on CTA, and 5 patients (5\%) had non-diagnostic image quality. In total, 16 patients (15\%) were immediately discharged after normal CTA and 90 patients (85\%) underwent invasive coronary angiography. Sensitivity, specificity, and positive and negative predictive values to detect significant CAD on CTA were 100, 87, 93, and $100 \%$, respectively. During mean follow-up of 13.7 months, no cardiovascular events occurred in patients with a normal CTA examination. In patients with non-significant CAD on CTA, no cardiac death or myocardial infarctions occurred and only 1 patient underwent revascularization due to unstable angina. In patients presenting with acute chest pain, an excellent clinical performance for the non-invasive assessment of significant CAD was demonstrated using CTA. Importantly, normal or non-significant CAD on CTA predicted a low rate of adverse cardiovascular events and favorable outcome during follow-up.

Keywords Acute coronary syndrome .

Multidetector computed tomography angiography . Coronary artery disease

\section{Introduction}

Every year, a substantial number of patients present at the emergency department with acute chest pain complaints [1]. While diagnosis is relatively 
straightforward in case of typical ECG changes and elevated biomarkers, a substantial number of patients present with both biomarkers and ECG that are either within normal limits or inconclusive. Accordingly, most patients will undergo extensive work-up including invasive coronary angiography to exclude coronary artery disease (CAD) as the cause of their symptoms to avoid inappropriate discharge. However, this approach leads to many unnecessary hospital admissions and is both time-consuming and expensive. Therefore, a non-invasive and rapid examination to establish or exclude CAD as the underlying cause of symptoms could substantially improve the clinical care of patients presenting with acute chest pain.

Several studies have suggested that computed tomography coronary angiography (CTA) may be of value in the diagnostic work-up in patients with acute chest pain in the emergency department [2-4]. Recently, a new generation of scanners has been introduced equipped with 320 detector rows of $0.5 \mathrm{~mm}$ wide, yielding a maximum of $16 \mathrm{~cm}$ craniocaudal coverage [5]. This design allows threedimensional volumetric whole-heart imaging in a single gantry rotation. Accordingly, a marked reduction in radiation dose is achieved by the elimination of oversampling or overranging, observed with helical scanning techniques [6]. In addition, the 320-row CTA system eliminates the problem of stair-step artifacts caused by inter-heartbeat variations as well as a reduction in cardiac motion artifacts. Furthermore, the temporal resolution has improved (175 ms using half reconstruction) resulting in superior image quality and accuracy for the detection of CAD [7, 8].

The performance of 320-row CTA in the evaluation of significant CAD in clinical practice in patients presenting with acute chest pain and the relation to outcome has not been previously reported. Therefore, the purpose of the current study was to evaluate the performance of 320-row CTA in the identification of significant $\mathrm{CAD}$ in patients presenting with acute chest pain and to examine the relation to outcome during follow-up.

\section{Methods}

The population consisted of patients included as part of an ongoing clinical registry who presented with acute chest pain to the Emergency Department. In all patients, physicians had sufficient clinical suspicion for an ischemic origin of chest pain and admitted these patients to the hospital to rule out presence of significant CAD $[9,10]$. However, patients presenting with an ST-segment elevation myocardial infarction (STEMI) were excluded and were immediately referred for direct percutaneous coronary intervention (PCI).

According to clinical protocol, patients were referred for CTA imaging for non-invasive evaluation of acute chest pain. Consequently, patients were referred for invasive coronary angiography (ICA) based on clinical presentation and/or imaging results to further evaluate the extent and severity of CAD. Due to the relative novelty of the use of CTA in patients with acute chest pain, a conservative approach was applied before discharging patients after CTA examination. If CTA examination showed no significant $\mathrm{CAD}$ and was of good to reasonable image quality and was in line with clinical presentation and/or biomarkers, patients were subsequently discharged from the hospital. The remaining patients (abnormal CTA, uninterpretable CTA or high clinical suspicion of CAD) were referred for ICA, which served as the standard of reference. In addition, TIMI risk scores were calculated and patients were classified as low, intermediate or high risk [11].

Exclusion criteria for CTA examination were: (1) (supra) ventricular arrhythmias and/or increased heart rate, (2) renal failure (glomerular filtration rate $<30 \mathrm{~mL} / \mathrm{min}$ ), (3) known allergy to iodine contrast material, (4) severe claustrophobia, (5) pregnancy, (6) previous coronary artery bypass grafting (CABG), (7) contra-indications for beta-blockers, (8) clinically unstable presentation and (9) STEMI.

CTA data acquisition

Prior to CTA examination, beta-blocking medication (metoprolol 50 or $100 \mathrm{mg}$, single oral dose, $1 \mathrm{~h}$ prior to CTA examination) was administered if the heart rate was $\geq 65$ beats per minute, unless contra-indicated. If heart rate was still $\geq 65$ beats per minute on arrival to the scanner and if no medical contra-indications existed, intravenous metoprolol (2.5-10 mg) was added. In addition, sublingual nitroglycerin ( 0.4 or $0.8 \mathrm{mg}$ sublingual) was administered $5 \mathrm{~min}$ prior to start scan. In all patients CTA was performed using a 
320-row CTA scanner (Aquilion ONE, Toshiba Medical Systems, Otawara, Japan) with 320 detector rows (each $0.50 \mathrm{~mm}$ wide) before ICA. The entire heart was imaged in a single volume, with a maximum of $16 \mathrm{~cm}$ cranio-caudal coverage, using prospective ECG triggering. If the heart rate was stable and $<60$ beats/min the phase window was set at $70-80 \%$ of R-R interval, if the heart rate was $60-65$ beats/min the phase window was set at $65-85 \%$ of R-R interval and if the heart rate was $\geq 65$ beats/min the phase window was set at $30-80 \%$ of the R-R interval (using multiple beats). Tube voltage and current were adapted to body mass index (BMI). Tube voltage was $100 \mathrm{kV}$ (BMI < $23 \mathrm{~kg} / \mathrm{m}^{2}$ ), $120 \mathrm{kV}$ (BMI, 23-35 kg/m²), or $135 \mathrm{kV}$ $\left(\mathrm{BMI} \geq 35 \mathrm{~kg} / \mathrm{m}^{2}\right)$ and maximal tube current was 400-580 mA (depending on body weight). Contrast material was administered in a triple-phase protocol: first a bolus of 60-80 ml, followed by $40 \mathrm{ml}$ of a 50:50 mixture of contrast and saline, followed by saline flush with a flow rate of $5-6 \mathrm{ml} / \mathrm{s}$ (Iomeron $400^{\circledR}$ ). Automatic bolus arrival detection was used to synchronize arrival of the contrast in the left ventricle with a threshold of +180 Hounsfield Units. All images were acquired during an inspiratory breath-hold of approximately $5 \mathrm{~s}$. First, a data set was reconstructed in the end-diastolic phase ( $75 \%$ of R-R interval) with a slice thickness of $0.50 \mathrm{~mm}$ and a reconstruction interval of $0.25 \mathrm{~mm}$. If motion artifacts were present, multiple phases were reconstructed to obtain maximal diagnostic image quality. Total time for the CTA examination was typically $10-15 \mathrm{~min}$. Data sets were transferred to a remote workstation (Vitrea FX 1.0, Vital Images, Minnetonka, MN, USA). Radiation dose was quantified with a dose-length product conversion factor of $0.014 \mathrm{mSv} /(\mathrm{mGy} \times \mathrm{cm})$. When scanning prospectively at $70-80 \%$ of $\mathrm{R}-\mathrm{R}$ interval, estimated mean radiation dose was $3.6 \pm 0.9 \mathrm{mSv}$. When scanning prospectively at $65-85 \%$ of $\mathrm{R}-\mathrm{R}$ interval, estimated mean radiation dose was $6.0 \pm$ $1.7 \mathrm{mSv}$. The estimated mean radiation dose was $12.0 \pm 4.5 \mathrm{mSv}$ when scanning prospectively with multiple beats.

\section{CTA image analysis}

Assessment of the contrast-enhanced CTA datasets for the presence of significant CAD was performed by 2 experienced investigators. CTA examinations were assessed as recommended by the SCCT guidelines for the interpretation and reporting of CTA [12]. Image quality was scored as good, reasonable, moderate or non-diagnostic [13]. Coronary anatomy was assessed in a standardized manner by dividing the coronary artery tree into 17 segments according to a modified American Heart Association (AHA) classification [14]. Each segment was deemed interpretable or uninterpretable, and evaluated for the presence of $\geq 50 \%$ luminal narrowing on the axial slices with the assistance of multiplanar and curved multiplanar reconstructed images. Subsequently, vessel-based analysis was performed. In the analysis on a vessel basis, the left main was considered part of the left anterior descending coronary artery (LAD) and the intermediate branch was considered part of the left circumflex coronary artery (LCx). Of note, if one segment was uninterpretable, an intention to diagnose strategy was applied. However, if more than one segment in a single vessel was uninterpretable, the vessel was considered to be of non-diagnostic image quality. Finally, a patient-based analysis was performed using a similar approach. Each CTA was classified according to three groups: normal, nonsignificant $\mathrm{CAD}(<50 \%$ luminal narrowing $)$ and significant $\mathrm{CAD}$ ( $\geq 50 \%$ luminal narrowing). If one vessel was uninterpretable, an intention to diagnose strategy was applied. However, if more than one vessel was uninterpretable, the entire scan was considered to be of non-diagnostic image quality.

Invasive coronary angiography

ICA was performed according to standard protocols. Quantitative coronary angiography (QCA) analysis was performed on a segment basis by an observer unaware of CTA findings with the use of validated QCA software (QAngioXA 6.0, CA-CMS, Medis Medical Imaging Systems, Leiden, The Netherlands). Coronary artery segments by QCA were also evaluated using a 17-segment AHA coronary tree model. The tip of the catheter was used for calibration and for each segment examined, the reference diameter and minimum luminal diameter were measured and percent diameter stenosis was reported. Measurements were performed on at least two orthogonal projections and the highest percent diameter stenosis was used for further analysis. Significant CAD was defined as $\geq 50 \%$ luminal narrowing on QCA analysis. 
Follow-up

Revascularization procedures (percutaneous coronary intervention (PCI) and/or CABG) during hospitalization were recorded. After discharge, patient follow-up data were gathered from the departmental Cardiology Information system by a single observer blinded to the baseline CTA and ICA results using clinical visits or contacted by standardized telephone interviews. The following cardiovascular events were regarded as clinical endpoints: cardiac death, non-fatal myocardial infarction, and unstable angina requiring revascularization. Cardiac death was defined as death by acute myocardial infarction, ventricular arrhythmias, or refractory heart failure. Non-fatal infarction was defined based on criteria of typical chest pain, elevated cardiac enzyme levels, and typical changes on the ECG [15]. Unstable angina was defined according to the European Society of Cardiology guidelines as acute chest pain with or without the presence of ECG abnormalities, and negative cardiac enzyme levels [9].

\section{Statistical analysis}

First, the performance (sensitivity, specificity, positive and negative predictive values including $95 \%$ confidence intervals) of CTA for the detection of significant $\mathrm{CAD}$ (defined as luminal narrowing $\geq 50 \%$ on QCA) was calculated on patient, vessel and segment basis. ICA was the standard of reference for detection of significant $\mathrm{CAD}$ and a patient, vessel or segment was classified as true positive if significant CAD was identified correctly by CTA. Initially, the performance of 320-row CTA was determined excluding patients, vessels and segments of non-diagnostic image quality. Subsequently, a second analysis was performed in which non-diagnostic patients, vessels and segments were included in the analysis and were considered positive for significant CAD. Clinical events were reported as numbers and percentages according to three groups: normal CTA, non-significant CAD on CTA $(<50 \%$ luminal narrowing $)$ and significant CAD on CTA ( $\geq 50 \%$ luminal narrowing). Statistical analysis was performed using SPSS 17.0 software (SPSS Inc., Chicago. Illinois).

\section{Results}

Patient population

In total, 204 patients with a primary complaint of acute chest pain were found eligible during the inclusion period. Exclusion criteria were present in 98 patients $(48 \%)$ (clinical instability $(n=25)$, impaired renal function $(n=16)$, previous $\mathrm{CABG}$ $(n=15)$, (supra) ventricular arrhythmias and/or increased heart rate $(n=9)$, scanner availability $(n=6)$, contra-indications to beta-blockers $(n=3)$ and other $(n=24)$ (Fig. 1$)$. The remaining study population consisted of 106 patients who underwent non-invasive coronary angiography with a 320-row CTA scanner. Baseline patient characteristics are described in Table 1. In summary, mean age was $57 \pm 10$ years and 71 patients were male $(67 \%)$. The majority of patients $(83 \%)$ had a low to intermediate TIMI risk score.

CTA

Overall, image quality was good in 50 patients (47\%), reasonable in 40 patients $(38 \%)$ and moderate in 11 patients $(10 \%)$. Five patients $(5 \%)$ had a non-diagnostic CTA examination. Furthermore, 23 patients $(22 \%)$ had a normal CTA, 19 patients (18\%) had nonsignificant CAD on CTA, 59 patients $(55 \%)$ had significant $\mathrm{CAD}$ on CTA, and the remaining 5 patients (5\%) with non-diagnostic scan quality were considered as significant CAD on CTA. In 16 patients (15\%) with a normal CTA examination, clinical presentation and biomarkers were in line with the CTA findings and therefore patients were discharged home (case example illustrated in Fig. 2). Nevertheless, the remaining 7 patients with a normal CTA examination had a high clinical suspicion of CAD and they were still referred for ICA. In total, 90 patients $(85 \%)$ were clinically referred for ICA (case example illustrated in Fig. 3).

Patient based analysis

When excluding patients with non-diagnostic scan quality, CTA correctly identified the presence of significant CAD in all 55 patients $(100 \%)$. Furthermore, CTA correctly excluded significant CAD in 26 of 30 patients (87\%). Thus, only 4 patients were 
Fig. 1 Flow chart of patient inclusion. CTA indicates computed tomography coronary angiography; ICA, invasive coronary angiography; $\mathrm{CV}$ events, cardiovascular events

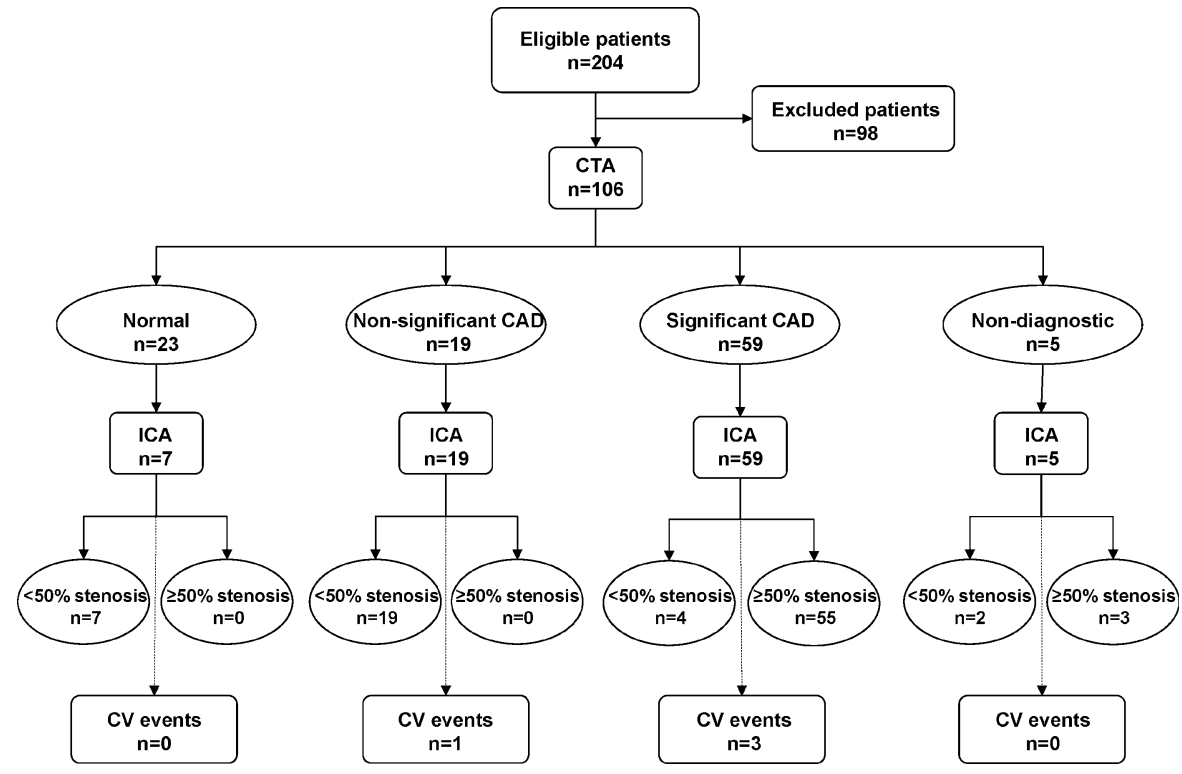

overestimated on CTA. Importantly, no patients with significant $\mathrm{CAD}$ on ICA were missed by CTA. Accordingly, when excluding non-diagnostic CTA examinations, sensitivity and specificity on a patient's basis were 100 and $87 \%$, respectively. Moreover, when including non-diagnostic CTA examinations (considered as positive for the presence of significant CAD), sensitivity and specificity on a patient basis were 100 and $81 \%$, respectively (Table 2).

Vessel analysis

Out of the 255 vessels (85 patients) evaluated on CTA, 6 vessels (2\%) (right coronary artery (RCA), $n=5$ and LAD, $n=1$ ) were deemed non-diagnostic. Regarding the vessels with diagnostic image quality, 93 of 94 vessels were correctly identified by CTA as significant CAD on ICA. Additionally, 147 of 155 vessels were correctly identified as normal or non-significant CAD by CTA. However, 1 vessel which was deemed as significant CAD on ICA was incorrectly classified as non-significant CAD on CTA. Moreover, CTA overestimated 8 vessels as significant $\mathrm{CAD}$ which were classified as non-significant CAD on ICA. Thus, when excluding nondiagnostic vessels from analysis, sensitivity and specificity on a vessel basis were 99 and 95\%, respectively. However, when including non-diagnostic vessels, sensitivity and specificity on a vessel basis were 99 and 92\%, respectively (Table 2).
Segment analysis

In total, 44 of 1,216 segments $(4 \%)$ were deemed non-diagnostic on CTA examination. Of the 44 segments, 21 segments were located in the RCA, 15 segments were located in the LAD and 8 segments were located in the LCx. Out of the 1,172 segments with diagnostic image quality, significant $\mathrm{CAD}$ was correctly identified by CTA in 136 of the 149 segments. Moreover, CTA correctly ruled out presence of significant CAD in 989 of 1,023 segments. Nevertheless, CTA overestimated 34 lesions that were considered as non-significant CAD on ICA. In addition, 13 lesions were underestimated on CTA which were deemed as significant CAD on ICA. Accordingly, when excluding non-diagnostic segments, the sensitivity and specificity for the detection of significant CAD on a segment basis were 91 and $97 \%$, respectively. Notably, when including nondiagnostic segments, the sensitivity and specificity for the detection of significant CAD on a segment basis were 91 and 93\%, respectively (Table 2).

\section{Revascularization during admission period}

In relationship to CTA findings, in the 7 patients with normal CTA, no revascularization was performed. Of the 19 patients with non-significant CAD on CTA examination, PCI was performed in 2 patients $(11 \%)$, both with angiographically non-significant $\mathrm{CAD}$. One 
Table 1 Baseline patient characteristics

\begin{tabular}{ll}
\hline Number of patients & 106 \\
Age & $57 \pm 10$ \\
Male gender & $71(67 \%)$ \\
Cardiovascular risk factors & \\
Hypertension $^{\mathrm{a}}$ & $55(52 \%)$ \\
Hypercholesterolemia $^{\mathrm{b}}$ & $41(39 \%)$ \\
Family history of CAD $_{\text {Current smoker }}$ & $54(51 \%)$ \\
Diabetes & $41(39 \%)$ \\
Obesity ( $\left.\geq 30 \mathrm{~kg} / \mathrm{m}^{2}\right)$ & $17(16 \%)$ \\
Medication at time of referral & $29(27 \%)$ \\
Beta-blockers & \\
Statins & $50(47 \%)$ \\
Aspirine & $52(49 \%)$ \\
ACE-inhibitors & $52(49 \%)$ \\
Previous myocardial infarction & $45(43 \%)$ \\
Previous PCI & $28(26 \%)$ \\
Mean troponin level ( $\mu \mathrm{g} / \mathrm{L})$ & $32(30 \%)$ \\
TIMI score & $0.05 \pm 0.16$ \\
Low & \\
Intermediate & $36(34 \%)$ \\
High & $52(49 \%)$ \\
Average heart rate during CTA & $18(17 \%)$ \\
\hline Dat a & $58 \pm 8$ \\
\hline
\end{tabular}

Data are absolute values, percentages or means \pm standard deviation

$C A D$ coronary artery disease, $A C E$ angiotensin converting enzyme, $P C I$ percutaneous coronary intervention, TIMI thrombolysis in myocardial infarction, $Q C A$ quantitative coronary angiography, CTA computed tomography angiography

a Defined as systolic blood pressure $\geq 140 \mathrm{~mm} \mathrm{Hg}$ or diastolic blood pressure $\geq 90 \mathrm{~mm} \mathrm{Hg}$ or the use of antihypertensive medication

b Serum total cholesterol $\geq 230 \mathrm{mg} / \mathrm{dL}$ or serum triglycerides $\geq 200 \mathrm{mg} / \mathrm{dL}$ or treatment with lipid lowering drugs

patient underwent PCI with stent placement due to coronary spasm and 1 patient underwent PCI because of angiographically non-significant lesion which was deemed significant on intravascular ultrasound. In the 59 patients with significant CAD on CTA examination, PCI was performed in 42 patients (71\%), CABG was performed in 6 patients $(10 \%)$ and 7 patients $(12 \%)$ were treated conservatively. Lastly, in 5 patients with non-diagnostic image quality, 3 patients had significant CAD on ICA, PCI was performed in 1 patient $(20 \%)$, CABG was performed in
1 patient (20\%) and 1 patient (20\%) was treated conservatively.

Clinical end points during follow-up

The mean follow-up period was 13.7 months (25-75th percentile: 6.5-18.7 months). The overall cardiovascular event rate was low (3.8\%), only 4 cardiovascular events occurred in all patients. Of note, 1 patient with non-significant CAD on CTA and normal coronary arteries on ICA died of a noncardiac cause 12 days after the angiographic procedure as a result of the consequences of severe chronic pulmonary hypertension which developed after previous liver transplantation. Importantly, no cardiovascular events occurred in the 23 patients with a normal CTA examination. In the 19 patients with non-significant CAD on CTA, no cardiac death or myocardial infarctions occurred and only 1 patient $(5.3 \%)$ was hospitalized because of unstable angina and underwent revascularization for a borderline lesion in the LAD. Moreover, in the 59 patients with significant CAD on CTA, 1 patient $(1.7 \%)$ underwent non-fatal myocardial infarction and 2 patients $(3.4 \%)$ were revascularized because of unstable angina. Lastly, in the 5 patients with a non-diagnostic CTA no cardiovascular events occurred.

\section{Discussion}

Several studies have recently shown a high sensitivity and specificity of 320-row CTA for the detection of significant $\mathrm{CAD}$ in patients electively referred for ICA [7, 8, 16]. However, to the best of our knowledge, the clinical performance of the 320-row scanner in patients presenting with acute chest pain has not been previously reported. Therefore, the purpose was to evaluate the performance of 320-row CTA in the identification of significant CAD in patients presenting with acute chest pain and to assess clinical outcome.

In summary, 16 patients were discharged after normal CTA without further invasive examination. In the remaining subset of patients with acute chest pain referred for ICA, an excellent sensitivity and specificity of 100 and $87 \%$ for the detection of significant CAD using CTA was demonstrated when excluding 

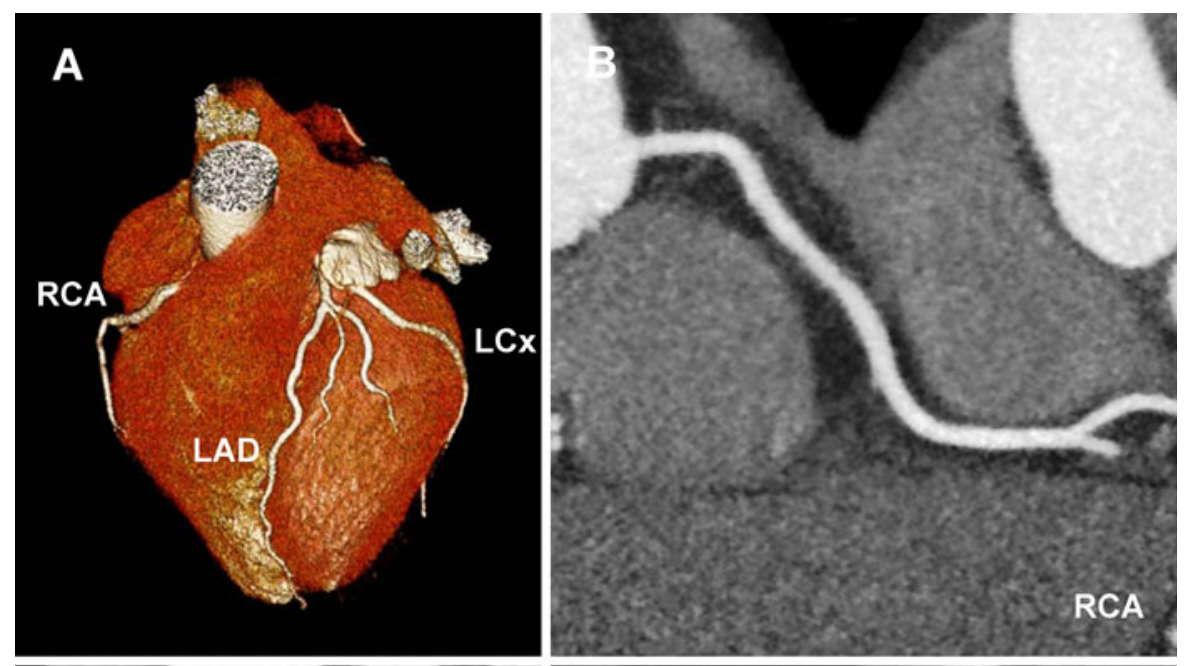

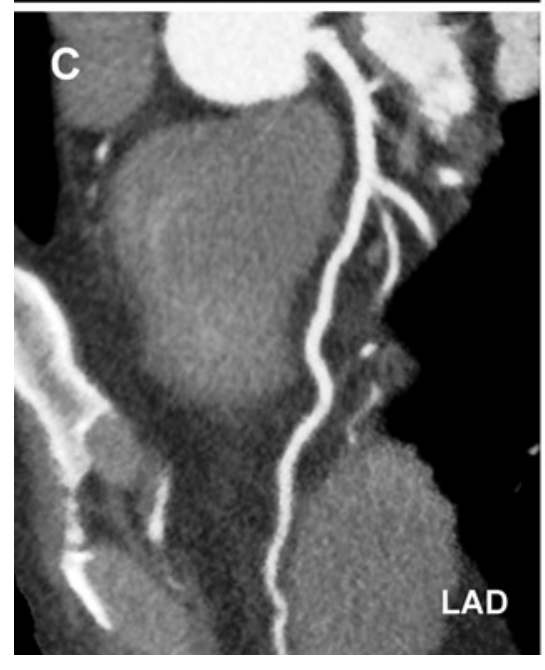

Fig. 2 Non-invasive coronary angiography using 320-row computed tomography angiography (CTA) of a 42-year-old male presenting with acute chest pain revealing a normal CTA examination. The patient was subsequently discharged home and no events occurred during follow-up. a A three-dimensional volume-rendered reconstruction of the heart, providing

scans with non-diagnostic image quality. In addition, a negative predictive value of $100 \%$ was observed, indicating that 320-row CTA did not miss any patients with significant CAD. When including CTA scans of non-diagnostic image quality, sensitivity and negative predictive value remained high (100\%), but specificity decreased to $81 \%$. In all patients with a normal CTA, no cardiovascular events occurred in the follow-up period. The excellent negative predictive value of 320-row CTA suggests that this technique could be useful in ruling out CAD in patients presenting with acute chest pain.

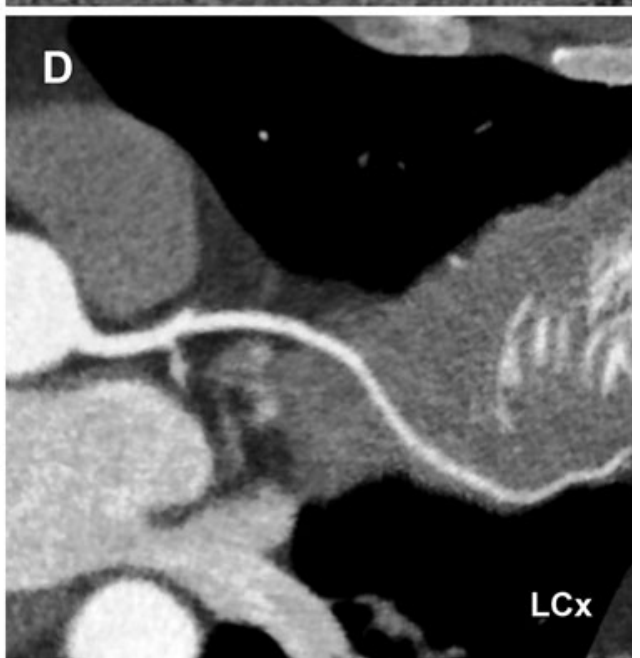

an overview of the left anterior descending coronary artery $(L A D)$ and proximal right coronary artery $(R C A)$. b-d The curved multiplanar reconstructions of a normal RCA, LAD, and left circumflex coronary artery (LCx), respectively, without significant coronary artery disease

Nevertheless, as demonstrated by the relatively low specificity values, lesion severity is still being overestimated by CTA as compared to ICA.

The present findings seem to be in line with previous studies evaluating the diagnostic accuracy with 64-row CTA for the detection of the presence of coronary stenosis in patients presenting with acute chest pain [2-4, 17, 18]. Recently, Chow et al. assessed the diagnostic accuracy of 64-row CTA in 107 patients with acute chest pain as compared to ICA [19]. The investigators demonstrated a good diagnostic accuracy of 64-row CTA for detection of significant CAD 

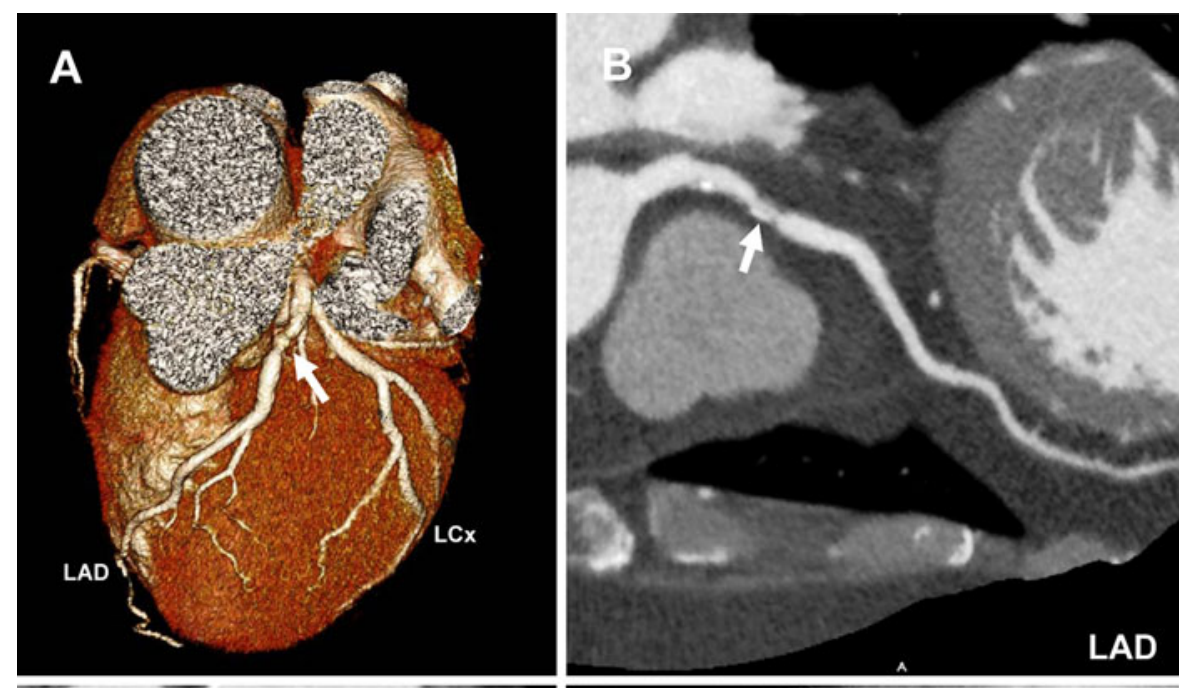

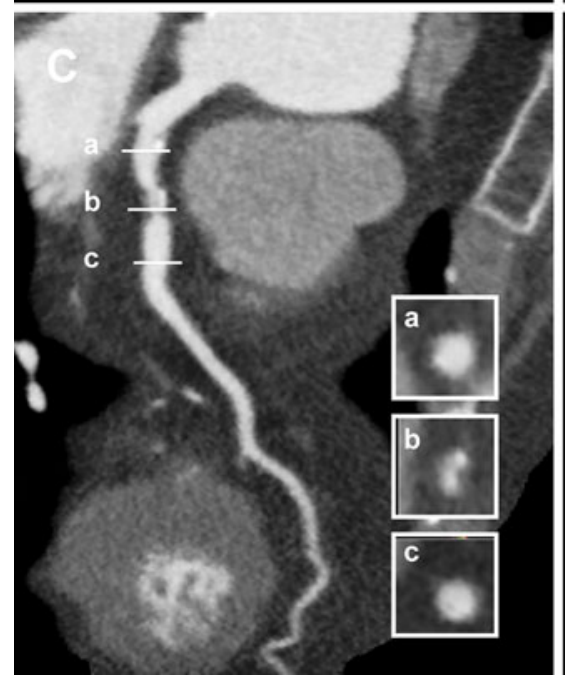

Fig. 3 Non-invasive coronary angiography using 320-row computed tomography angiography of a 68-year-old female presenting with acute chest pain revealing a significant lesion in the mid left anterior descending coronary artery $(L A D)$. a $\mathrm{A}$ three-dimensional volume-rendered reconstruction of the heart, providing an overview of the LAD and left circumflex coronary

(defined as $\geq 50 \%$ luminal narrowing), reporting sensitivity, specificity, and positive and negative predictive values on a patient basis of $94,90,89$, and $94 \%$, respectively. Similarly, Meijboom et al. also reported a high diagnostic accuracy of 64-row CTA for detecting significant CAD in 104 patients with non-ST elevation myocardial infarction [20]. In line with our findings, the investigators reported an excellent sensitivity and negative predictive value of $100 \%$. However, specificity was also relatively low (75\%). These studies suggest that CTA could be an attractive non-

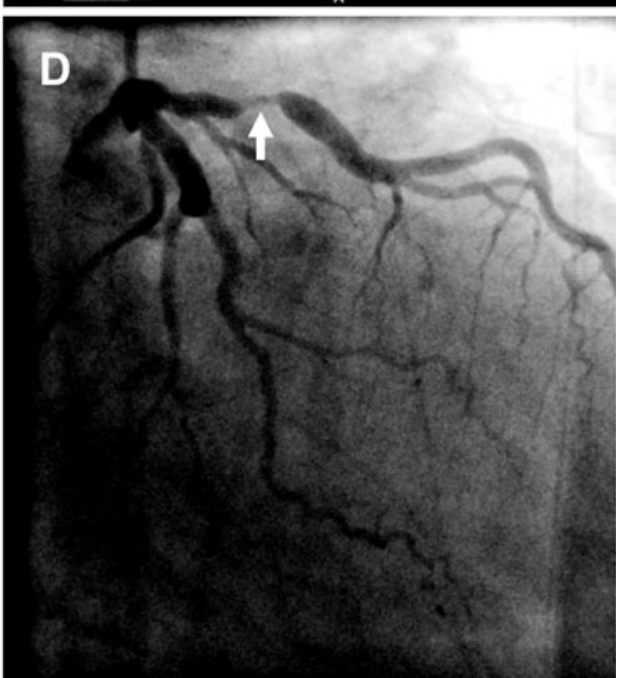

artery (LCx) revealing signs of luminal narrowing in the mid LAD (arrow). b, c Curved multiplanar reconstruction of the LAD demonstrating a significant stenosis in the mid LAD (arrow and b, respectively). d Corresponding invasive coronary angiography image confirming the presence of a significant stenosis in the mid LAD (arrow)

invasive modality to exclude CAD in patients presenting with acute chest pain.

Regarding the performance of 320-row CTA, Dewey et al. recently assessed the diagnostic accuracy of 320-row CTA in 30 patients with stable chest pain as compared to ICA [8]. Besides significantly reducing radiation dose, the investigators demonstrated a good diagnostic accuracy of 320-row for detection of significant CAD (defined as $\geq 50 \%$ luminal narrowing), reporting sensitivity, specificity, and positive and negative predictive values on a 
Table 2 Diagnostic performance of 320-row computed tomography angiography for detection of significant coronary artery disease in patients presenting with acute chest pain, excluding and including non-diagnostic segments, vessels and patients

\begin{tabular}{lccc}
\hline & Segment analysis & Vessel analysis & Patient analysis \\
\hline Excluding non-diagnostic segments, vessels and patients & & \\
Sensitivity & $136 / 149(91 \%, 87-96 \%)$ & $93 / 94(99 \%, 97-100 \%)$ & $55 / 55(100 \%)$ \\
Specificity & $989 / 1,023(97 \%, 96-98 \%)$ & $147 / 155(95 \%, 91-98 \%)$ & $26 / 30(87 \%, 75 \%-99 \%)$ \\
PPV & $136 / 170(80 \%, 74-86 \%)$ & $93 / 101(92 \%, 87-97 \%)$ & $55 / 59(93 \%, 87-99.6 \%)$ \\
NPV & $989 / 1002(99 \%, 98-99 \%)$ & $147 / 148(99 \%, 98-100 \%)$ & $26 / 26(100 \%)$ \\
Diagnostic accuracy & $1125 / 1172(95 \%, 95-97 \%)$ & $240 / 249(96 \%, 94-99 \%)$ & $81 / 85(95 \%, 91-99.7 \%)$ \\
Including non-diagnostic segments, vessels and patients & & $5 / 90(6 \%)$ \\
Non-diagnostic & $44 / 1216(4 \%)$ & $6 / 255(2 \%)$ & $58 / 58(100 \%)$ \\
Sensitivity & $138 / 151(91 \%, 87-96 \%)$ & $95 / 96(99 \%, 97-100 \%)$ & $26 / 32(81 \%, 68-95 \%)$ \\
Specificity & $989 / 1065(93 \%, 91-94 \%)$ & $147 / 159(92 \%, 88-97 \%)$ & $58 / 64(91 \%, 83-98 \%)$ \\
PPV & $138 / 214(64 \%, 58-71 \%)$ & $95 / 107(89 \%, 83-95 \%)$ & $26 / 26(100 \%)$ \\
NPV & $989 / 1002(99 \%, 98-99 \%)$ & $147 / 148(99 \%, 98-100 \%)$ & $84 / 90(93 \%, 88-98 \%)$ \\
Diagnostic accuracy & $1127 / 1216(93 \%, 91-94 \%)$ & $242 / 255(95 \%, 92-98 \%)$ & \\
\hline
\end{tabular}

Data are absolute values used to calculate percentages. Data in parentheses are percentages with 95\% confidence intervals. Patients with scans of non-diagnostic image quality were excluded from vessel and segment analyses

$N P V$ negative predictive value, $P P V$ positive predictive value

patient basis of 100,94, 100, and 92\%, respectively. Similarly, de Graaf et al. demonstrated a high diagnostic accuracy of 320-row CTA for detection of significant CAD in 64 patients referred for ICA [7]. As compared to the older generation 64-row CTA scanners, one of the main advantages of the 320-row CTA system is the improved $z$-axis coverage of $16 \mathrm{~cm}$ that can cover the entire heart in a single gantry rotation. Therefore, 320-row CTA can accurately acquire images of the heart in a single heartbeat, which is substantially faster than the 6-10 s needed for 64-row CTA. Accordingly, a decreased amount of contrast is needed and breath hold is reduced to a minimum. Furthermore, due to the volumetric scanning approach, the presence of stair-step artifacts and typical pitch artifacts are eliminated. Lastly, as the entire heart can be imaged in one rotation, there is potential to assess myocardial perfusion as part of the acute chest pain work-up. These advantages of volumetric scanning may substantially optimize image quality and could possibly expand the use of CTA.

The excellent negative predictive value of CTA has made it a particular interesting modality for rapid diagnosis of patients with acute chest pain. Importantly, patients with a normal CTA had an excellent clinical outcome without cardiovascular events during the follow-up period. Therefore, the present findings demonstrate that the strength of CTA is that it can completely and safely rule out presence of CAD. Nevertheless, events still occurred in patients with significant as well as non-significant CAD on CTA, indicating that presence of plaque on CTA may still be considered relevant in this patient population. Notably, in the present study, the overall cardiovascular event rate was low $(3.8 \%)$. However, the majority of patients (both low and high risk) underwent subsequent intervention after CTA, including revascularization and initiation of anti-atherosclerotic medical treatment, which may have had a positive effect on outcome.

In line with the present study, several studies have demonstrated that coronary CTA is useful and safe in ruling out $\mathrm{CAD}$ and facilitates early and accurate release of patients with acute chest pain $[3,4,18]$. Rubinshtein and colleagues prospectively studied 58 patients with acute chest pain in the emergency department and evaluated the performance of 64-row coronary CTA for diagnosing or excluding acute coronary syndrome [4]. The investigators evaluated clinical outcomes during a follow-up of 15 months and found that no deaths or myocardial infarctions occurred in the 35 patients discharged from the emergency department with a normal CTA. In a 
larger cohort, Hollander et al. prospectively evaluated 586 low to intermediate risk patients who received 64-row CTA in the emergency department for evaluation of acute chest pain [18]. Interestingly, patients discharged from the emergency department with a negative CTA $(n=476,84 \%)$ had a very low event rate of $0.2 \%(n=1)$. These studies demonstrate that CTA has a high positive predictive value for diagnosing an acute coronary syndrome, and a normal CTA predicts a favourable outcome and low rate of major adverse cardiovascular events during follow-up. Furthermore, CTA evaluation in patients with acute chest pain has been shown to significantly reduce time to diagnosis, lower costs and require fewer repeat investigations when compared to standard of care [2]. As a result, the noninvasive assessment of coronary anatomy and presence of significant stenosis with 320-row CTA may impact clinical management in patients presenting with acute chest pain.

\section{Clinical implications}

In the current study, patients with acute chest pain and a normal CTA examination had an excellent clinical outcome without cardiovascular events during the follow-up period. Therefore, it seems that patients presenting with acute chest pain and a normal CTA can be safely discharged. Furthermore, although CTA still overestimates lesion severity, most patients with significant CAD on CTA (81\%) were subsequently revascularized by means of PCI or CABG. Thus, 320-row CTA was demonstrated to be a relatively safe and useful technique for both excluding and including CAD in patients with acute chest pain. Nevertheless, it is important to realize that the presence of significant CAD on CTA does not necessarily equal myocardial ischemia, unless microcirculatory flow is impeded. Indeed, anatomical assessment of the coronaries is most reassuring when the vessels are normal or have minimal disease. In addition, even though 320-row CTA significantly reduces radiation dose and contrast dose, careful patient selection regarding age, renal function and body mass index are of fundamental importance to optimize use of CTA. Furthermore, heart rate reduction is essential for acquiring diagnostic image quality and is usually more challenging in patients admitted to the emergency department. Currently, use of CTA in symptomatic patients has a class IIa recommendation for patients with an intermediate pre-test likelihood of obstructive disease, suggesting that this technique can be an appropriate alternative for the evaluation of patients with acute chest pain $[21,22]$.

\section{Limitations}

The following limitations of the present study should be considered. First, in the present study a referral bias is present as patients are referred for ICA on the basis of CTA examination findings in combination with clinical presentation and/or other imaging results. Nevertheless, this approach reflects current clinical practice and thus could possibly be valuable in evaluating the use of this new imaging technique. Secondly, no quantitative measurements were performed on segments assessed with 320-row CTA, such as percentage luminal narrowing. Although visual estimation will be sufficient in most segments, more precise grading of luminal narrowing is preferred. However, new developments are ongoing, and dedicated software techniques are being expected. Furthermore, a substantial number of patients with acute chest pain were excluded such as patients with hemodynamic or electrical instability, or ongoing chest pain to prevent further delays of revascularization treatment. Therefore, coronary CTA is not generally applicable to all patients with acute chest pain. In addition, the presence of a significant stenosis on coronary $\mathrm{CT}$ does not by definition confirm the presence of ACS or significant flow limitation. Potentially, a combination of anatomical CTA images with functional information would be preferable. Finally, radiation dose still remains of concern. Nevertheless, with the new 320-row systems as well as prospective triggering, radiation dose may be even lower than estimated radiation dose with conventional ICA $[8,23]$.

\section{Conclusion}

The present study shows that 320-row CTA enables accurate and safe evaluation of significant CAD in patients presenting with acute chest pain. Importantly, a negative CTA predicted a low rate of adverse cardiovascular events and favorable outcome during follow-up. Consequently, the noninvasive 
assessment of coronary anatomy and presence of significant CAD with 320-row CTA may impact clinical management in patients presenting with acute chest pain.

Acknowledgments This work was supported by the Dutch Heart Foundation (The Hague, The Netherlands) [grant number 2007B223 to J.E.V.] and the Dutch Technology Foundation STW (Utrecht, the Netherlands), applied science division of I and the Technology Program of the Ministry of Economic Affairs [grant number 10084 to F.R.G.]. J.J.B. has research grants from BMS medical imaging (New York, United States) and GE Healthcare (Chalfont St Giles, United Kingdom).

\section{Conflict of interest None.}

Open Access This article is distributed under the terms of the Creative Commons Attribution Noncommercial License which permits any noncommercial use, distribution, and reproduction in any medium, provided the original author(s) and source are credited.

\section{References}

1. Pitts SR, Niska RW, Xu J et al (2008) National hospital ambulatory medical care survey: 2006 emergency department summary. Natl Health Stat Report 7:1-38

2. Goldstein JA, Gallagher MJ, O'Neill WW et al (2007) A randomized controlled trial of multi-slice coronary computed tomography for evaluation of acute chest pain. J Am Coll Cardiol 49(8):863-871

3. Hoffmann U, Bamberg F, Chae CU et al (2009) Coronary computed tomography angiography for early triage of patients with acute chest pain: the ROMICAT (Rule Out Myocardial Infarction using Computer Assisted Tomography) trial. J Am Coll Cardiol 53(18):1642-1650

4. Rubinshtein R, Halon DA, Gaspar T et al (2007) Usefulness of 64-slice cardiac computed tomographic angiography for diagnosing acute coronary syndromes and predicting clinical outcome in emergency department patients with chest pain of uncertain origin. Circulation 115(13):1762-1768

5. Rybicki FJ, Otero HJ, Steigner ML et al (2008) Initial evaluation of coronary images from 320-detector row computed tomography. Int J Cardiovasc Imaging 24(5):535-546

6. Hausleiter J, Meyer T, Hermann F et al (2009) Estimated radiation dose associated with cardiac $\mathrm{CT}$ angiography. JAMA 301(5):500-507

7. De Graaf FR, Schuijf JD, Van Velzen JE et al (2010) Diagnostic accuracy of 320-row multidetector computed tomography coronary angiography in the non-invasive evaluation of significant coronary artery disease. Eur Heart J 31(15):1908-1915

8. Dewey M, Zimmermann E, Deissenrieder F et al (2009) Noninvasive coronary angiography by 320-row computed tomography with lower radiation exposure and maintained diagnostic accuracy: comparison of results with cardiac catheterization in a head-to-head pilot investigation. Circulation 120(10):867-875

9. Bassand JP, Hamm CW, Ardissino D et al (2007) Guidelines for the diagnosis and treatment of non-ST-segment elevation acute coronary syndromes. Eur Heart $\mathbf{J}$ 28(13):1598-1660

10. Anderson JL, Adams CD, Antman EM et al (2007) ACC/ AHA 2007 guidelines for the management of patients with unstable angina/non-ST-elevation myocardial infarction: a report of the American College of Cardiology/American Heart Association Task Force on Practice Guidelines (Writing Committee to Revise the 2002 Guidelines for the Management of Patients With Unstable Angina/Non-STElevation Myocardial Infarction) developed in collaboration with the American College of Emergency Physicians, the Society for Cardiovascular Angiography and Interventions, and the Society of Thoracic Surgeons endorsed by the American Association of Cardiovascular and Pulmonary Rehabilitation and the Society for Academic Emergency Medicine. J Am Coll Cardiol 50(7):e1-e157

11. Antman EM, Cohen M, Bernink PJ et al (2000) The TIMI risk score for unstable angina/non-ST elevation MI: a method for prognostication and therapeutic decision making. JAMA 284(7):835-842

12. Raff GL, Abidov A, Achenbach S et al (2009) SCCT guidelines for the interpretation and reporting of coronary computed tomographic angiography. J Cardiovasc Comput Tomogr 3(2):122-136

13. Shuman WP, Branch KR, May JM et al (2008) Prospective versus retrospective ECG gating for 64-detector CT of the coronary arteries: comparison of image quality and patient radiation dose. Radiology 248(2):431-437

14. Austen WG, Edwards JE, Frye RL et al. (1975) A reporting system on patients evaluated for coronary artery disease. Report of the Ad Hoc Committee for Grading of Coronary Artery Disease, Council on Cardiovascular Surgery, American Heart Association. Circulation 51(4 Suppl):5-40

15. Thygesen K, Alpert JS, White HD (2007) Universal definition of myocardial infarction. Eur Heart J 28(20):25252538

16. De Graaf FR, Schuijf JD, Van Velzen JE et al (2010) Diagnostic accuracy of 320-row multidetector computed tomography coronary angiography to noninvasively assess in-stent restenosis. Invest Radiol 45(6):331-340

17. Hoffmann U, Nagurney JT, Moselewski F et al (2006) Coronary multidetector computed tomography in the assessment of patients with acute chest pain. Circulation 114(21):2251-2260

18. Hollander JE, Chang AM, Shofer FS et al (2009) One-year outcomes following coronary computerized tomographic angiography for evaluation of emergency department patients with potential acute coronary syndrome. Acad Emerg Med 16(8):693-698

19. Chow BJ, Joseph P, Yam Y et al (2010) Usefulness of computed tomographic coronary angiography in patients with acute chest pain with and without high-risk features. Am J Cardiol 106(4):463-469

20. Meijboom WB, Mollet NR, van Mieghem CA et al (2007) 64-Slice CT coronary angiography in patients with non-ST elevation acute coronary syndrome. Heart 93(11):13861392 
21. Taylor AJ, Cerqueira M, Hodgson JM et al. (2010) ACCF/ SCCT/ACR/AHA/ASE/ASNC/NASCI/SCAI/SCMR 2010 appropriate use criteria for cardiac computed tomography: a report of the American College of Cardiology Foundation Appropriate Use Criteria Task Force, the Society of Cardiovascular Computed Tomography, the American College of Radiology, the American Heart Association, the American Society of Echocardiography, the American Society of Nuclear Cardiology, the North American Society for Cardiovascular Imaging, the Society for Cardiovascular Angiography and Interventions, and the Society for Cardiovascular Magnetic Resonance. J Am Coll Cardiol 56(22):1864-1894

22. Wijns W, Kolh P, Danchin N et al (2010) Guidelines on myocardial revascularization: the task force on myocardial revascularization of the European Society of Cardiology (ESC) and the European Association for Cardio-Thoracic Surgery (EACTS). Eur Heart J 31(20):2501-2555

23. Herzog BA, Wyss CA, Husmann L et al (2009) First headto-head comparison of effective radiation dose from lowdose 64-slice CT with prospective ECG-triggering versus invasive coronary angiography. Heart 95(20):1656-1661 